\title{
Myeloproliferative Neoplasms: An Overview
}

\section{KATHLEEN FINNEGAN}

Clin Lab Sci 2011;24(3):178

Kathleen Finnegan, MS, MT(ASCP)SH ${ }^{C M}$, Stony Brook University Health Science Center, School of Health Technology and Management, Stony Brook, NY

Address for Correspondence: Kathleen Finnegan, MS, $M T(A S C P) S H^{C M}$, Chair, Department of Clinical Laboratory Sciences, Stony Brook University Health Science Center, School of Health Technology and Management, Level 2, Room 442, Stony Brook, NY 11794-8205, 631444-3224, Kathleen.finnegan@stonybrook.edu

\section{INTRODUCTION}

The myleproliferative neoplasms (MPNs) are a closely related group of clonal stem cell hematologic blood disorders characterized by an increase in one or more cell lines of the bone marrow. The proliferation of red blood cells (RBCs), white blood cells (WBCs) or platelets is usually mature in morphology. The MPNs have overlapping clinical features but different etiologies. The original name of these disorders was the myeloid proliferative disorders or MPDs proposed by William Damashek in $1951 ;^{1}$ they were renamed by the World Health Organization (WHO) in 2008 to "myeloproliferative neoplasms"2 due to new findings. The 2008 WHO classification of MPNs has added new entities, including chronic myelogenous leukemia, Philadelphia chromosome-positive (CML); chronic neutrophilic leukemia (CNL); polycythemia vera (PV); primary myelofibrosis (PMF); essential thrombocythemia (ET); chronic eosinophilic leukemia, not otherwise specified (CEL/NOS); mastocytosis; and myeloproliferative neoplasm, unclassifiable (MPN-U). The identification of numerous molecular mutations such as the "breakpoint cluster region/Abelson fusion 1 $(B C R / A B L 1)$ mutation, Janus kinase 2 (JAK2) mutation, (JAK2), myeloproliferative leukemia virus oncogene homolog $(M P L)$ and others, , has contributed to the further understanding of the genetic complexity of the MPNs. The current classification of the MPNs is based on clinical presentation, pathologic descriptions, histological information, and genetic mutations. The genetic mutations or molecular markers have become essential for diagnosis, prognosis and treatment of these disorders.

The MPNs are characterized by a hypercellular bone marrow due to unregulated cell proliferation. Usually one cell line is increased, but all may be elevated. The classification is based on which cell line is affected in the bone marrow and peripheral blood. The MPNs are more commonly seen in middle-aged adults, ages 40 50, with an additional preponderance after age 60 . They are rarely seen in a pediatric population. See Table 1 for a summary of each entity with its main characteristics.

Myeloproliferative neoplasms have no known cause. Symptoms, clinical presentation, prognosis and progression vary depending on the patient's age, health status and MPN category. Some patients may have a slowly progressing disease that may not require any treatment beyond careful monitoring, while others may progress to more advanced stages and others may terminate as an acute leukemia.

In summary, the MPNs are involved with one or more of the following features: overproduction of one or more cell lines, hypercellular marrow or bone marrow fibrosis, cytogenetic abnormalities, point mutations, extramedullary hematopoiesis, hemorrhagic bleeding and or thrombosis and can transform into an acute leukemia.

\section{Chronic Myelogenous Leukemia (CML)}

The most predominant feature of CML is severe leukocytosis with the entire spectrum of myeloid cell 
FOCUS: UPDATE ON MYELOPROLIFERATIVE NEOPLASMS

Table 1: 2008 WHO Classification of Myeloproliferative Neoplasms

Disorder

Chronic myelogenous leukemia (CML), $\mathrm{BCR} / \mathrm{ABL} 1$ positive

Chronic neutrophilic leukemia (CNL)

Polycythemia vera (PV)

Primary myelofibrosis (PMF)

Essential thrombocythemia (ET)

Chronic eosinophilic leukemia, not otherwise specified (CEL/NOS)

Mastocytosis

Myeloproliferative neoplasm, unclassified

\section{Characteristics}

- Abnormal pluripotential bone marrow stem cell

- Associated with the BCR/ABL1 fusion gene

- Increased WBC count, myeloid cells in all stages of granulocytic development

- $\quad<3 \%$ monocytes

- $\quad<2 \%$ myeloblasts

- Absolute basophilia

- Peripheral blood neutrophilia; neutrophilic precursors account for less than $5 \%$

- Myeloblasts not observed in peripheral blood

- Bone marrow hypercellular

- BCR/ABL1 negative

- Elevated RBC, WBC, and platelet count

- $\quad$ BCR/ABL1 negative

- $\quad>95 \%$ exhibit JAK2 mutation2

- WBC count is variable

- Elevated platelet count

- Bone marrow proliferation; megakaryocytes and granulocytes predominate

- Extramedullary hematopoiesis

- Fibrosis of the bone marrow

- BCR/ABL1 negative

- $50 \%$ exhibit JAK2 mutation2

- Thrombocytosis, > 450 X 109/L, often over $1,000 \times 109 / \mathrm{L}$

- $\quad$ BCR/ABL1 negative

- $\quad 40-50 \%$ exhibit JAK2 mutation2

- Clonal proliferation of eosinophil precursors

- Eosinophil count greater than $1.5 \times 10^{9} / \mathrm{L}$

- Increased eosinophils in peripheral, bone marrow and tissues

- $<20 \%$ blasts in the bone marrow

- Presences of multifocal compact clusters of abnormal mast cells in one or more organs

- $80 \%$ of cases have skin involvement

- Only applied to cases that have definite features of MPN but fail to meet the criteria of other MPN

- Features overlap two or more MPN development appearing in the peripheral blood. The Philadelphia chromosome characterizes CML; a reciprocal translocation between the long arms of chromosome 9 and 22 referred to as $\mathrm{t}(9 ; 22)$. This cytogenetic translocation leads to the formation of the
$B C R / A B L 1$ mutation, which is the cause of unregulated hematopoiesis due to unregulated tyrosine kinase activity. ${ }^{3}$ Normal tyrosine kinase regulates metabolic pathways, controls apoptosis and confines cells to the bone marrow until they have matured. With the 


\section{FOCUS: UPDATE ON MYELOPROLIFERATIVE NEOPLASMS}

increase of tyrosine kinase activity there is a loss of apoptosis, loss of bone marrow adhesion with an increased release of myeloid cells that leave the bone marrow too soon. The presence of the $B C R / A B L 1$ rearrangement is considered diagnostic when present with the clinical manifestations of CML. ${ }^{4}$ Peripheral blood findings show an increase in neutrophils. There are various stages of neutrophil maturation with peaks in percent of myelocytes and segmented neutrophils. ${ }^{2}$ Basophilia and eosinophilia are common. The monocyte count comprises less than $3 \%$ of the differential. The blast count can be 1-3\%. As CML evolves and the blast count rises, the disease can enter an aggressive or accelerated phase and can terminate in an acute phase or blast crisis when the blast count is greater than $20 \%$.

\section{Chronic Neutrophilic Leukemia (CNL)}

CNL is characterized by a slowly progressive neutrophilic leukocytosis in the peripheral blood and bone marrow. Peripheral blood findings reveal neutrophilia with a WBC count greater than $25 \mathrm{x}$ $10^{9} / \mathrm{L}^{2}$ The granulocytes usually appear mature and consist of bands and segmented neutrophils. The neutrophils can display toxic changes but do not show dysplasia (maturation abnormalities). The younger stages of the neutrophil account for less than $5 \%$ of the WBCs. The RBCs and platelets appear to be normal. The patients present with hepatosplenomegaly, and are negative for the Philadelphia chromosome/BCR/ABL1 mutation. CNL affects adults over 60 years of age. Diagnosis is made after the exclusion of a reactive neutrophilia or other MPNs. Myelodysplasia signals transformation to acute myeloblastic leukemia (AML). Patients survive 6 months to more than 20 years. ${ }^{2}$

\section{Polycythemia Vera (PV)}

PV is a clonal stem cell malignancy of the bone marrow. The primary defect involves a pluripotential stem cell capable of differentiating into RBCs, granulocytes, and platelets. There is an increase in $\mathrm{RBC}$ volume with an elevated WBC and platelet count. Absolute erythrocytosis is the most outstanding feature. The $\mathrm{RBC}$ count can range from $6-10 \times 10^{9} / \mathrm{L}$. PV has a prevalence ranging from 0.7 to 2.3 per 100,000 population. ${ }^{2}$ It is a chronic disease seen predominantly in adults over 60 years of age. The JAK2 or JAK2V617 mutation is found in approximately $95 \%$ of patients. ${ }^{5}$ The JAK2 enzymes play a role in normal blood development and immune function. These enzymes, members of the tyrosine kinase family, mediate signal transduction in cell pathways that regulate cell growth and cell survival. ${ }^{6}$ When JAK2 signaling is dysregulated it may result in increased myeloid cell survival, differentiation and proliferation. The defect in cell death creates an accumulation of cells.

The onset of the disease is gradual. Most symptoms are related to the increased erythrocyte mass, which causes cardiovascular disease, hyperviscosity of the blood and enlarged spleen. Common complications seen with this disorder include thrombotic events and bleeding tendencies. Treatment includes therapeutic phlebotomy to keep the hematocrit below $45 \%$ and myelosuppressive cytoreduction to reduce the hematopoietic cells.

\section{Primary Myelofibrosis (PMF)}

PMF is characterized by ineffective erythropoiesis and abnormal megakaryocyte production. It is a clonal hematopoietic stem cell disorder that is accompanied by marrow fibrosis, leukoerythroblastosis, splenomegaly and extramedullary hematopoiesis. As the bone marrow becomes more fibrotic and normal hematopoiesis can no longer take place, extramedullary hematopoiesis occurs in the liver and spleen, enlarging both organs. The WBC and platelet counts are elevated initially but decrease as the disease progresses. The peripheral blood smear presents with a leukoerythroblastic picture, which includes teardrop RBCs, poikilocytosis, anisocytosis, nucleated red blood cells, and immature myeloid cells. Approximately $50 \%$ of patients have been found to have the $J A K 2 \mathrm{~V} 617 \mathrm{~F}$ mutation. ${ }^{7}$ The onset is gradual. Clinical findings include progressive anemia, splenomegaly, weakness, weight loss, and pain in the extremities and in the left upper quadrant. PMF must be distinguished from other MPNs since the findings of PMF are similar to CML and PV in the latter stages.

\section{Essential Thrombocythemia (ET)}

ET is characterized by an uncontrolled clonal proliferation of megakaryocytes in the bone marrow with a sustained peripheral blood platelet count of more than $450 \times 10^{9} / \mathrm{L}^{2}$ This increase in the platelet 


\section{FOCUS: UPDATE ON MYELOPROLIFERATIVE NEOPLASMS}

peripheral blood count has been associated with bone marrow megakaryocyte hyperplasia and the increased risk of thrombotic and hemorrhagic complications. In this disorder the JAK2 $\mathrm{V} 617 \mathrm{~F}$ or MPL W515K/L mutation are positive for $60 \%$ to $70 \%$ of ET cases. ${ }^{8}$ Bone marrow assessment and molecular testing are key to the diagnosis of ET. The bone marrow cellularity is normal or slightly increased, but there is an abundance of large, mature-appearing megakaryocytes without morphological abnormalities. In peripheral blood the platelet diameter ranges from $(<1 \mathrm{um}$ to $>8 \mathrm{um})$ with atypical forms. Sometimes platelets may be giant and bizarre in shape. The WBC count and differential are usually normal. Most patients are asymptomatic at presentation with markedly increased platelet counts, but symptoms may include headache, dizziness, weakness and slurred speech. ET is prevalent in patients 50-60 years of age. Diagnosis of ET requires the exclusion of reactive thrombocytosis and of the other MPNs. Current therapeutic approaches are focused on reducing the amount of platelets and preventing thrombotic complications. Plateletpheresis reduces the platelet count but it is not long term. Chemotherapy such as hydroxyurea can be used in combination with plateletpheresis to reduce thrombohemorrhage complications.

\section{Chronic Eosinophilic Leukemia, Not Otherwise Specified (CEL/NOS)}

CEL/NOS is a clonal proliferation of eosinophils in peripheral blood, bone marrow and tissues. Patients who have a persistent absolute eosinophil count of at least $1.5 \times 10^{9} / \mathrm{L}$ are classified in this CEL/NOS group. ${ }^{2}$ Exclusion of reactive eosinophilia must be ruled out for diagnosis. The morphology of the eosinophil may show few granules with clear areas of cytoplasm and cytoplasmic vacuolization. The nucleus can be hypersegmented or hyposegmented. Anemia and thrombocytopenia may be present. Many patients with nonclonal forms of eosinophilia fall within the category of "idiopathic" hypereosinophilia; this is defined as an eosinophilia that has persisted for longer than six months and an underlying cause cannot be determined. No specific cytogenetic or molecular abnormality has been determined. Cases with platelet-derived growth factor rearrangement $\mathrm{A}(P D G F R A)$ or $\mathrm{B}(P D G F R B)$ are excluded from this category.

\section{Mastocytosis}

Mast cell disease is characterized by tissue infiltration by multifocal aggregates of abnormal mast cells. The disease is classified into cutaneous mastocytosis (CM) where the infiltrates are within the skin, and systemic mastocytosis (SM) involving extracutaneous organs and bone marrow. SM may present with or without skin lesion involvement. Systemic mastocytosis is characterized by a somatic mutation of the KIT protooncogene. KIT codes for the tyrosine kinase receptor that is involved in the regulation of mast cell development. The KIT mutation is detected in virtually all patients with SM. ${ }^{9}$ The diagnosis of mastocytosis should exhibit multifocal clusters of aggregates of mast cells in the bone marrow. Mastocytosis can occur at any age. $\mathrm{CM}$ is more common in children. SM is seen more frequently in adults.

\section{CONCLUSION}

As molecular genotyping becomes a more integral part of the diagnostic criteria of the MPNs, treatment guidelines and prognostic data will improve patient management. Recent advances and the better understanding of the molecular mutations have improved the outcome of MPNs. Important in the clinical management of these disorders is the reduction of thrombohemorrhagic complications, disease symptoms and the minimization of risk of transformation to AML or other malignancies. The precise diagnosis of MPNs still remains a challenge.

\section{REFERENCES}

1. Dameshek W. Some speculations on the myloproliferative syndromes, Blood. 1951;6:372-5.

2. Swerdlow S, Campo E, Harris L, et al. WHO classification of tumors of haematopoietic and lymphoid tissues, International Agency for Research on Cancer, Lyon. 2008;14-15,32-65.

3. Spivak, J. Chronic Myeloproliferative Disorders. http://cme. medscape.com/viewarticle/551491 Accessed on 7/7/2010.

4. Druker BJ, Sawyers CL, Kantarjian H, et al. Activity of a specific inhibitor of the $B C R-A B L$ tyrosine kinase in blast crisis of chronic myeloid leukemia and acute lymphoblastic leukemia with the Philadelphia chromosome. N Engl J Med. 2001; 344:1038-42.

5. Delhommeu F, Jeziorowska D, Marzac C, Casadevall N, Molecular aspects of myeloproliferative neoplasms, Int J Hematol. 2010;91:165-73.

6. Levine RL, Pardanani A,Tefferi A, Gilliland DG. Role of $J A K 2$ in the pathogenesis and therapy of myeloproliferative disorders. Nat Rev Cancer. 2007;7:673-83. 


\section{FOCUS: UPDATE ON MYELOPROLIFERATIVE NEOPLASMS}

7. Tefferi A, Thiele J, Vardiman JW. The 2008 World Health Organization classification system for myeloproliferative neoplasms: order out of chaos. Cancer. 2009;9:3842-7.

8. Thiele J, Kvaanicka HM. The WHO diagnostic criteria for polycythemia vera, essential thrombocythemia and primary myelofibrosis. Curr Hematol Malig. 2009;4:33-40.
9. Vannucchi AM, Gugliclmelli P, Teffer A, American Cancer Society advances in understanding and management of myeloproliferative neoplasms. CA Cancer J Clin. 2009;59: 171-91.

The Focus section seeks to publish relevant and timely continuing education for clinical laboratory practitioners. Section editors, topics, and authors are selected in advance to cover current areas of interest in each discipline. Readers can obtain continuing education credit (CE) through P.A.C.E.' by completing the continuing education registration form, recording answers to the examination, and mailing a photocopy of it with the appropriate fee to the address designated on the form. Suggestions for future Focus topics and authors, and manuscripts appropriate for CE credit are encouraged. Direct all inquiries to the Clin Lab Sci Editorial Office, Westminster Publishers, 315 Westminster Court, Brandon MS 39047. (601) 214-5028, (202) 315-5843 (fax).westminsterpublishers@comcast.net. 